\title{
Inhalt 1985
}

\section{Seeber, S., Leverkusen}

Grundlagen der hochdosierten Cytarabinosid-Behand-

lung 4

Gassmann, W.; Schmitz, N. und Löffler, H., Kiel

Ergebnisse der hochdosierten Cytarabin-Therapie. - Eine

Übersicht 8

Meusers, P.; Heidemann, H.; Lunscken, C. ;

Uppenkamp, M. und Brittinger, G., Essen; Zou, P.,

Wuhan/VR China

Hochdosierte Cytarabin-Behandlung: Erfolgverspre-

chende Therapiemodalität bei resistenten akuten myelo-

ischen Leukämien im Rezidiv 16

Kuse, R. und Küchler, R., Hamburg

Nicht-hämatologische Toxizität bei der hochdosierten

Cytarabin-Therapie 20

Buchbesprechungen 23

Nowrousian, M. R.; Seeber, S.; Miller, A. A.;

Anders, Ch.; Öhl, S. und Schmidt, C. G., Essen

Hochdosierte Cytarabin-Behandlung bei akuten Leuk

ämien und Meningiosis Leucaemica: Klinik und Pharma-

kokinetik 26

Jehn, U., München

Erfahrungen mit der HD-Ara C Behandlung bei

Leukämie-Patienten 37

Brudler, O.; Heil, G. und Kurrle, E., Ulm Hochdosis-Cytosinarabinosid in der Behandlung

rezidi-vierter oder refraktärer akuter myeloischer Leukämien. . 39

Dickerhoff, R. und Haas, R. J., München

Therapieerfahrungen mit hochdosiertem ARA-C und

L-ASP 44

Neuigkeiten für die klinische Praxis 46

Pralle, H. und Breithaupt, H., Gießen

Ergebnisse der Therapie mit hochdosiertem Cytosinarabinosid 48

Pees, H. W. und Radtke, H. , Homburg

Hochdosierte Cytarabinbehandlung mit Asparaginase

(CAPIZZI-Schema) bei rezidivierter oder refraktärer

AML des Erwachsenen 53

Kuse, R. und Küchler, R., Hamburg

Diskussionsbeitrag zum Rundtischgespräch über hoch- 
dosiertes Cytarabin 56

Seeber, S., Leverkusen

Hochdosiertes Cytosinarabinosid. - Eine Zusammen-

fassung $\quad 57$

Onkologie-Mitteilungen $\quad 60$

Editorial 68

Memorandum und Statut $\quad 71$

Kassenärztliche Bundesvereinigung $\quad 74$

Brenner, U.; Schindler, J.; Müller, J. M.; Keller, H. W. und Walter, M. , Köln

Plasmaaminosäurenmuster bei Karzinomen des Gastro-

intestinaltrakts 78

Klingler, W. und Daweke, H., Bochum

Familiäres Plasmozytom bei Mutter und Sohn

Sonderbände 87

Wander, H.-E.; Teichmann, A. T.; Cremer, P.;

Wieland, H.; Kruse, B.; Nagel, G. A.; Kuhn, W. und

Seidel, D., Göttingen

Veränderungen der Serumlipoproteine unter hormonel-

len Therapieformen des Mammakarzinoms und anderer

endokrin abhängiger Tumoren 88

Jehn, U., München und Zittoun, R., Paris/Frankreich

AML-6-Studie zum Wert einer zyklisch alternierenden

Chemotherapie während der Remission bei akuter mye

loischer Leukämie 94

Jehn, U. , München und Löwenberg, B. , Paris I Frankreich

AML-7-Studie zum Wert einer intensiven Remissions-

induktion bei alten Patienten mit akuter myeloischer

Leukämie $\quad 97$

Neuigkeiten für die klinische Praxis 99

Friedmann, W.; Steffens, J. und Lobeck, H., Berlin

Immunhistochemische Darstellung tumorassoziierter

Antigene bei Harnblasenkarzinomen mit mono- und

polyklonalen Antiseren

105

Manfreda, D.; Aigner, H.; Pflanzl, G. und

Redtenbacher, H., KlagenfurtlÖsterreich und

Daxenbichler, G., Innsbruck/Österreich

Vergleich zwischen biochemischer und histochemischer

Hormonrezeptorenbestimmung bei Mammakarzinomen Ill

Mross, K. und Rauschecker, H. , Göttingen und

Wolf rum, D. I., Bad Soden-Allendorf

Ergebnisse mit dem Tissue Polypeptide Antigen (TPA)

und Plasma-TPA-Werte im postoperativen Verlauf bei

primären Mammakarzinompatientinnen: Erste Ergeb

nisse einer Langzeitstudie 114

Onkologie-Mitteilungen 125

Buchbesprechungen 132 
Queißer, W., Mannheim

Richtlinien der AIO zur Abfassung von Protokollen der

Phase-П-Prüfung von Zytostatika 133

In memoriam Mildred Scheel 140

Mahmoud, H. K; Öhl, S.; Schaefer, U. W.; Beelen, D.;

Grosse-Wilde, H.; Doxiadis, L; Richter, H. J.

und Schmidt, C. G. , Essen

Behandlung der Haarzell-Leukämie mit rekombinantem

Leukozyten A Interferon 141

422

Jahres-Inhalt 1985

Gastl, G.; Denz, H.; Abbrederis, C; Huber, H.;

Troppmair, J.; Wiegele, J.; Niederwieser, D.; Flener, R. und Huber, C, Innsbruck!Österreich

Induktion kompletter Remissionen bei Haarzell-Leuk-

ämie durch Therapie mit nieder dosiertem humanen

Rekombinanteninterferon-Alpha-2-ARG 143

Ehninger, G. und Ostendorf, P., Tubingen; Ho, A. D.

und Seither, E. , Heidelberg; Meyer, P. und

Mjaaland, L, Würzburg

Mitoxantron in der Behandlung des Rezidivs oder thera-

pierefraktärer Leukämien 146

Freund, M.; Políwoda, H. und Eisert, R., Hannover; Bodenstein, H., Minden

Behandlung akuter myeloischer Leukämien mit einer

Kombination aus Daunorubicin, Cytosin-Arabinosid und

6-Thioguanin ohne Erhaltungstherapie 150

Urbanitz, D.; Pielken, H.-J.; Koch, P.; Büchner, Th.; Hiddemann, W.; Heinecke, A. und van de Loo, J., Münster; Wendt, F. und Maschmeier, G., Essen-Werden Immuntherapie der akuten myeloischen Leukämien mit Neuraminidase-behandelten allogeneischen Blasten; Ergebnisse einer prospektiven randomisierten Studie . . 157

Jehn, U., München; Zittoun, R., Paris/Frankreich

und Löwenberg, B. , Rotterdam/Holland

AML-6- und AML-7-Studie zur Behandlung der akuten

myeloischen Leukämie: Zyklisch alternierende Chemo-

therapie während der Remission bzw. Remissionsinduk-

tion und Überlebenszeit bei alten Menschen 160

Buchbesprechungen 165

Mittermüller, J.; Klob, H. J.; Gerhartz, H. H. undWilmanns, W., München

Zytotoxische Wirkung der niedrig dosierten Ara-C-The-rapie. - Untersuchungen bei einer

Patientin mit Leuk-ämierezidiv nach allogener Knochenmarktransplantation 168

Neuigkeiten fur die klinische Praxis 172

Göbel, U. undJürgens, H., Düsseldorf; Brandeis, W., Heidelberg; Havers, W., Essen; Gadner, H., WienlÖsterreich; Spaar, H.-J., Bremen Vorläufige Ergebnisse der GPO-Studie für nichttestikuläre Keimzelltumoren bei Kindern und Jugendlichen . . . 178 
Hiddemann, W.; Urbanitz, D.; Preusser, P.; Balleisen, L.

und Büchner, Th., Münster; Achterrath, W., Neu-Isenburg

AMSA/Etoposid (VP 16-213): Eine Phase-I/II-Studie bei

refraktärer akuter myeloischer Leukämie $\quad 181$

Sonderbände 184

Onkologie-Mitteilungen 185

Dunzendorfer, U. und Knöner, M., Frankfurt/M.

Therapie mit Inhibitoren der Polyaminbiosynthese beim refraktären Prostatakarzinom. - Eine experimentelle und klinische Studie 196

Ehninger, G. undHaag, C., Tubingen; Ruckle, H. und Wilms, K., Würzburg

Die Therapie der Peritonealkarzinose mit intraperitonea-

ler Gabe von cis-Diaminodichloroplatin und systemischer

Natrium thiosulfatprotektion. - Klinische Ergebnisse

einer Pilotstudie und Pharmakokinetik 202

Gatzemeier, U.; Hossfeld, D. K.; Radenbach, D. und Zschaber, R., Hamburg

Chemotherapie mit Mitomycin C, Vindesin und Ifosfa-

mid in der Behandlung des inoperablen nicht-kleinzelli-

gen Bronchialkarzinoms 208

Neuigkeiten für die klinische Praxis 213

Gerhartz, H. und Jehn, U., München

Granulozytäre Vorläuferzellen bei akuter Leukämie:

Vergleich zwei verschiedener Kulturmethoden 214

Buchbesprechungen 218

Herschbach, P.; Rosbund, A.-M. und

Brengelmann, J. C. , München

Probleme von Krebspatientinnen und Formen ihrer Be-

wältigung 219

Maier, U. und Holzner, J. H. , Wien

Stellenwert der Urotheldysplasien in makroskopisch nor-

maler Schleimhaut bei Patienten mit Blasentumoren. -

Ergebnis einer randomisierten Studie mit und ohne intra-

vesikaler Mitomycin-C-Rezidivprophylaxe 232

Lellé, R. J.; Heidenreich, W. und Peter, H. H., Hannover Lymphoide Zellen im Venenblut von

100 Patientinnen mit Mammakarzinom.-Eine Verlaufsbeobachtung . . . 241

Onkologie-Mitteilungen 246

Kreienberg, R. und Melchert, F. , Mainz

Neuentwicklungen auf dem Gebiet der Marker zur Dia-

gnostik und Überwachung des Ovarialkarzinoms 253

Schröck, R., München; Hafter, R.; Graeff, H. und Schmid, L., Oberstaufen

Die simultane Bestimmung von Ca 125 und D-Dimer im

Plasma und Aszites beim Ovarialkarzinom 260

Sonderbände 262

Schillinger, H., Freiburg i. Br.

Sonographie zur Früherkennung und zur Entscheidung

derDignität 264 
Rohde, U.; Becker, H.; Weigert, F. und Lindner, P., Passau

Computertomograrnm in der Diagnostik des Ovarial

karzinoms 272

Neuigkeiten für die klinische Praxis 276

Dietel, M. undArps, H., Hamburg

Morphologie und Wachstumsverhalten von Ovarial-

tumoren 278

Jahres-Inhalt 1985

423

Rundtischgespräch «Staging»

Leitung: H. Schmidt-Matthiesen (Frankfurt) 286

Meerpohl, H. G., Freiburg i. Br.

Prognosefaktoren des Ovarialkarzinoms 296

Bender, H. G., Düsseldorf

Die radikale Tumoroperation beim begrenzten Ovarial-

karzinom 306

Frischbier, H.-J. und Bahnsen, J., Hamburg

Die Strahlentherapie beim primär radikal operierten

Ovarialkarzinom $\quad 310$

Illiger, H. J.; del Valle, F. und Mühlenstedt, D., Oldenburg

Adjuvante Chemotherapie beim primär radikal operier

ten Ovarialkarzinom 316

Rundtischgespräch «Die Therapie begrenzter, maligner

Ovarialtumoren anhand exemplarischer Fälle»

Leitung: A. Pfleiderer (Freiburg) 324

Onkologie-Mitteilungen 332

Pickel, H. und Lahousen, M., Graz, Stettner, H., Klagenfurtl Österreich

Die operative Tumorreduktion beim ausgedehnten

Ovarialkarzinom $\quad 344$

Brunner, K. W.; Goldhirsch, A. und Greiner, R., Bern/

Schweiz

Die Chemotherapie als Primärtherapie beim nicht radikal

operierten Ovarialkarzinom 347

Teufel, G.; Meerpohl, H. G. und Pfleiderer, A., Freiburg

Kontrolle des Ansprechens von Ovarialkarzinomen auf

die zytostatische Therapie und Stellenwert der Second-

look-Operation $\quad 356$

Greiner, R.; Goldhirsch, A.; Dreher, E.; Locher, G.; Jeanneret, Ch.; Meister, A.; Mihic, N. und

Veraguth, P., Bernl Schweiz

Die Ganzabdomenbestrahlung nach Kombinationsche-motherapie und Second-look-Laparotomie bei der Behandlung des fortgeschrittenen Ovarialkarzinoms . . . 364

Kühnle, H., Göttingen und Hilfrich, J., Hannover

Chemotherapie des fortgeschrittenen Ovarialkarzinoms.

- Studien Göttingen-Hannover 374 
Goldhirsch, A.; Greiner, R.; Dreher, E.; Locher, G.; Davis, B. W.; Reinhard, J. -P.; Joss, R. und Brunner, K. W., Bernl Schweiz

Die Behandlung des Ovarialkarzinoms nach einem multimodalen Konzept: Remissionsinduktion mit Chemothe rapie - Hexa PAMP und PAMP Regime - gefolgt von einer Abdomenganzbestrahlung 383

Hinweise für Autoren 387

Sevelda, P.; Salzer, H.; Glitsch, E.; Karrer, K. und Österreichische Arbeitsgemeinschaft zur Therapie des Ovarialkarzinoms, WienlÖsterreich Ergebnisse der 1. Österreichischen Ovarialkarzinom-Studie: Prospektiv randomisierter Vergleich einer neuen sequentiellen Chemotherapie mit 2 Standard-Schemata bei 150 Patientinnen des Stadiums III und IV 388 Neuigkeiten für die klinische Praxis. 392 Franz, G. und Lietz, H. , Hamburg Ergebnisse der Hamburger Ovarialblastom-Studie .... 394 Rundtischgespräch «Folgetherapie bei kompletter oder partieller Remission» 398

M. Kaufmann, Heidelberg Allgemeinbetreuung und operative Therapie bei progredientem oder rezidivierendem Ovarialkarzinom 403 U. Bruntsch, Nürnberg

Sekundäre Chemotherapie beim fortgeschrittenen Ova rialkarzinom - neue Medikamente 410 W. Jonat, Hamburg

Hormontherapie bei progredientem und rezidivierendem Ovarialkarzinom 417

Sonderbände 420

Jahres-Inhalt1985 421

Autorenverzeichnis 1985 . 